\title{
Biomateriales que inducen la remineralización del esmalte dental y dentina.
}

\author{
Biomaterials that induce remineralization of the dentin and enamel surface.
}

\author{
Ian Michel Jiménez Rosas*
}

\section{RESUMEN}

Desde los sesentas, con la invención del vidrio bioactivo, los tratamientos de remineralización se han popularizado entre los cirujanos dentistas y su utilización es cada vez mayor; la remineralización, en conjunto con las adecuadas medidas de higiene preventiva, representa uno de los mejores abordajes mínimamente invasivos y a un costo comparativamente bajo. Este estudio documental tiene por objetivo establecer una mejor comprensión del uso clínico de los biomateriales que inducen la remineralización de la superficie del esmalte dental y dentina. Se realizó una exploración utilizando motores de búsqueda (bases de datos en PubMed, Medigraphic, y Science Direct). El proceso de localización de los estudios relevantes se efectuó introduciendo palabras clave como: silicatos de calcio, fosfopéptidos de caseína-fosfato de calcio amorfo, remineralización, esmalte y dentina, incluyéndose en el procedimiento artículos de antigüedad no superior a siete años, en español e inglés, publicados en revistas científicas aprobadas por pares.Actualmente, no es posible remineralizar del todo la estructura dentaria, por lo cual, en un futuro cercano, los esfuerzos de la odontología de remineralización deben apuntar al desarrollo de agentes biomiméticos inteligentes que restauren al cien por ciento la estructura dental perdida.

Palabras clave: Remineralización, esmalte dental, dentina.

\section{ABSTRACT}

Since the sixties, with the invention of bioactive glass, remineralization treatments have become popular among dental surgeons. Their usage is increasing; remineralization, in conjunction with appropriate preventive hygiene measures, represents one of the best minimally invasive treatments at a relatively low cost. This documentary study aims to establish a better understanding of the clinical use of biomaterials that induce remineralization of the surface of teeth enamel and dentin. A search was conducted using search engines (PubMed and Medigraphic databases, and Science Direct). The search process for the relevant studies was carried out by introducing keywords such as calcium silicates, phosphopeptides of amorphous calcium casein-phosphate, remineralization, enamel and dentin, including in the search articles no older than seven years in Spanish and English published in scientific reviewed journals. Currently, it is not possible to completely remineralize the dentary structure so, in the near future, remineralization dentistry efforts should aim to develop

Keywords: Remineralization, dental enamel, dentin.

\section{INTRODUCCIÓN}

E n la actualidad, el estudio e investigación de los biomateriales los han llevado a evolucionar y definirse como sustancias que, al ponerse en contacto con tejidos vivos, provocan un efecto positivo sobre los mismos, induciendo una respuesta biológica específica en la interfase material-tejido. Estos elementos son empleados en la protección del complejo dentinopulpar por su capacidade de generar tejido calcificado en la

\footnotetext{
* Cirujano dentista en Escuela Militar de Odontología. UDEFA, México.

Recibido: 21 de agosto de 2020. Aceptado: 13 de julio de 2021.
}

Citar como: Jiménez RIM. Biomateriales que inducen la remineralización del esmalte dental y dentina. Rev ADM. 2021; 78 (4): 195-204. https://dx.doi. org/10.35366/101073 
zona de contacto entre el material y el tejido dental, entre otras funciones. ${ }^{1}$

Entre las principales características que deben reunir dicho tipo de sustancias para su empleo se pueden encontrar ser biocompatibles, estériles, no solubles ni reabsorbibles, bactericidas, bacteriostáticos, no ser afectados ni contaminados por sangre, capaces de estimular la formación de dentina reparativa, la adhesión a la dentina y otros materiales de restauración, resistir las fuerzas de compresión y tracción, al igual que proveer un buen sellado contra bacterias y líquidos, además de evitar la filtración al encontrarse situados en un entorno húmedo.

Hoy en día, los compuestos basados en silicato de calcio son reconocidos por su biocompatibilidad y por ser inductores de tejidos mineralizados, sin embargo, sus propiedades mecánicas no siempre son las ideales. Uno de los objetivos principales de los fabricantes es desarrollar una mezcla basada en dicho silicato, con dotes superiores a las ya existentes, por ejemplo el mineral de trióxido agregado (MTA) en relación con tiempo de fraguado, características físicas y manipulación. El Biodentine ${ }^{\circledR}$ es un material de nueva generación basado en tal silicato, a fin de ser utilizado como un sustituto de la dentina dañada. Este producto reúne grandes cualidades mecánicas, siendo de fácil manejo y teniendo una excelente biocompatibilidad, lo que lo hace un componente indicado en múltiples restauraciones.

\section{ANTECEDENTES}

El campo de los biomateriales dentales ha sido ampliamente investigado y explotado durante las últimas décadas, tal es el caso de los productos con fundamento en hidróxido de calcio $\mathrm{Ca}(\mathrm{OH})_{2}$ que son utilizados desde hace aproximadamente cien años, debido a su comprobada capacidad para favorecer la formación de dentina reparadora, su biocompatibilidad, la protección pulpar que generan contra estímulos térmicos y eléctricos, además de tener cualidades antimicrobianas. ${ }^{1}$

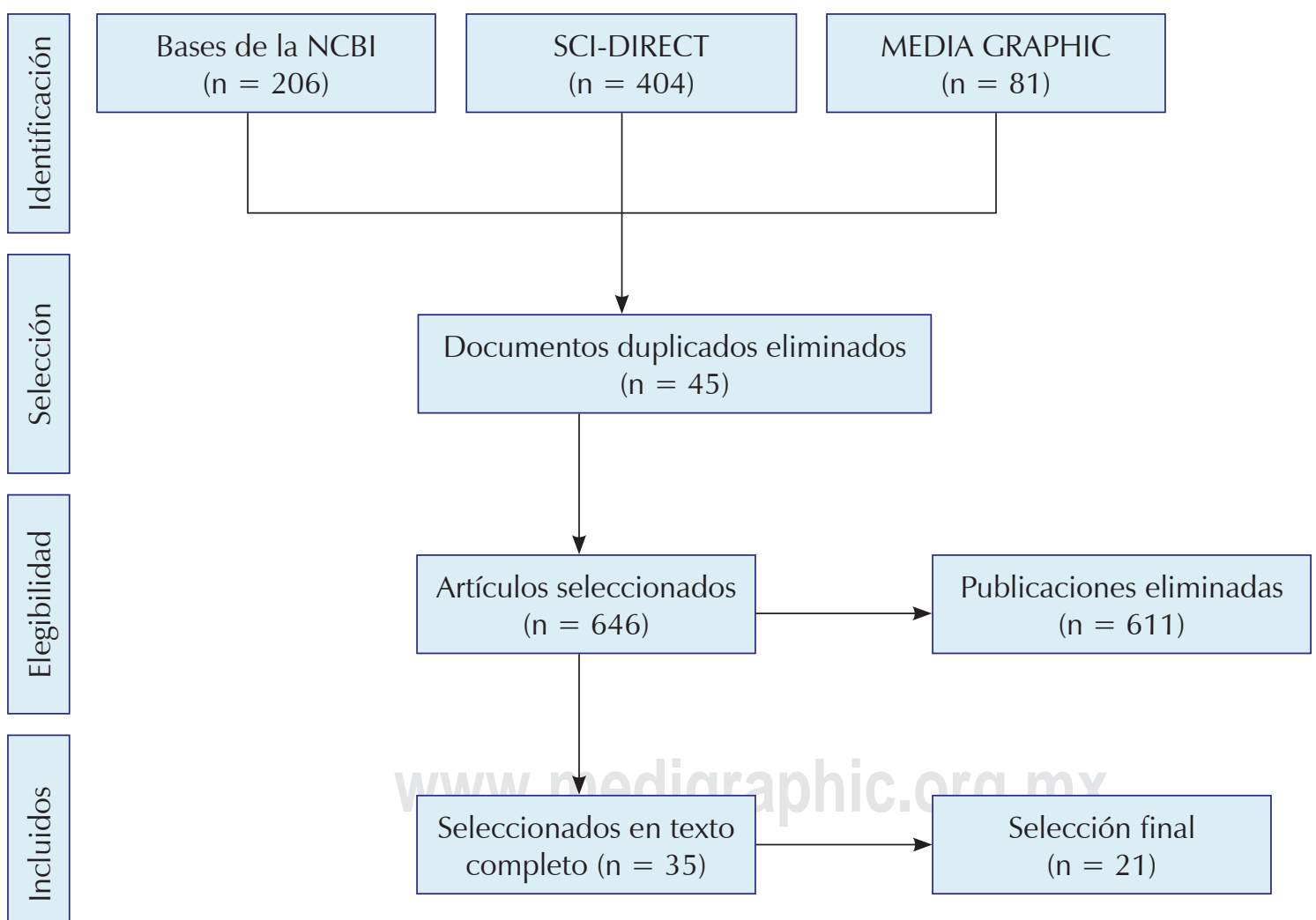

Fuente: directa.

Figura 1: Flujograma estrategia de búsqueda. 
Tabla 1: Selección final de los artículos de cementos basados con silicato de calcio. Los nueve documentos seleccionados tuvieron como objetivo evaluar la efectividad en bioactividad, inducción de precipitación y aumento en la densidad mineral.

\begin{tabular}{lll} 
Autores & Año & Revista \\
\hline Daneshpoor, et al. & 2020 & Journal of Clinical Experimental Dentistry \\
Salma Fathy & 2019 & Journal of Clinical Experimental Dentistry \\
Li, et al. & 2017 & The academy of dental materials (ELSEVIER) \\
Neves, et al. & 2018 & Clinical oral investigations \\
Noha Y Sadoon, et al. & 2020 & Brazilian Dental Journal \\
Pires, et al. & 2018 & Original Scientific Article \\
Schwendickk, et al. & 2019 & The academy of dental materials (ELSEVIER) \\
Suprastiwi, et al. & 2019 & Association of Support to Oral Health Research (APESB) \\
Trujillo, et al. & 2019 & International Journal of Dental Sciences \\
\hline
\end{tabular}

Fuente: directa.

Tabla 2: Descripción de artículos de materiales a base de fosfopéptidos de caseína-fosfato de

calcio amorfo. Los 12 artículos seleccionados tuvieron como objetivo evaluar la efectividad

en bioactividad, inducción de precipitación y aumento en la densidad mineral.

\begin{tabular}{lll} 
Autores & Año & Revista \\
\hline Zhang, et al. & 2018 & Scientific Reports \\
Vieira, et al. & 2017 & Journal of Applied Oral Science \\
Soares, et al. & 2017 & Journal of Clinical and Diagnostic Research \\
Thierens, et al. & 2019 & Journal of Applied Oral Science \\
Singh, et al. & 2016 & Progress in Orthodontics \\
Juárez, et al. & 2014 & Revista de Investigación Clínica \\
Al-Batayneh, et al. & 2017 & Archives of Oral Biology \\
Zheng, et al. & 2017 & Wear \\
Thabitha, et al. & 2016 & Pediatric Dental Journal \\
Elkassas, et al. & 2014 & Journal of Dentistry \\
Zhou, et al. & 2014 & Journal of Dentistry \\
Mohd, et al. & 2016 & International Journal of Paediatric Dentistry \\
\hline
\end{tabular}

Un estudio menciona al mineral de trióxido agregado, reportado por primera vez en el año de 1993 por Lee y asociados en los Estados Unidos, a partir del cemento Portland, con el cual comparte los componentes fundamentales como calcio, fosfato y sílice, el que recibió su aprobación por la Administración de Drogas y Alimentos de Estados Unidos (FDA) en 1998, mismo año en que comenzó a comercializarse. ${ }^{2}$ En el año 2008, la compañía francesa Septodont Ltd. con sede en SaintMaur-des-Fossés, Francia, desarrolló un nuevo material denominado Biodentine ${ }^{\circledR}$, el cual fue basado en la purificación del silicato de calcio, con características superiores a los compuestos ya existentes en relación al lapso de fraguado, propiedades mecánicas y de manipulación. Por su parte, la empresa Bisco en el año 2010 lanza al mercado el mencionado silicato modificado con resina conocida con las siglas (SCMR) o mejor conocido por su nombre comercial TheraCal $\mathrm{LC}^{\circledR}$ sustancia fotopolimerizable. Actualmente, estos ingredientes son empleados en la protección del complejo dentino-pulpar. ${ }^{3}$ 


\section{SILICATO DE CALCIO}

Los cementos a base de silicato de calcio se introdujeron por primera vez en la odontología en el año 1993, cuando Torabinejad inventó una fórmula basada en cemento Portland buscando producir el agregado de trióxido mineral, o MTA gris. Este material estaba compuesto principalmente de silicato tricálcico, silicato dicálcico, aluminato tricálcico y aluminoferrito tetracálcico, además de sulfato cálcico y óxido de bismuto. En el año 2002, se desarrolló una versión blanca de MTA, que era idéntica a la forma gris, pero carecía de aluminoferrito tetracálcico y tenía niveles reducidos de aluminato.

Desde su introducción, los MTA se han utilizado sobre todo en aplicaciones de endodoncia, como la reparación de raíces perforadas, apexificación o recubrimiento de pulpa, debido a sus tiempos de trabajo y fraguado relativamente largos. Septodont (Saint-Maur-
des-Fossés, Francia) introdujo Biodentine ${ }^{\circledR}$, un cemento dental de fraguado rápido con fundamento en el citado silicato (de aquí en adelante, tal nombre comercial se utilizará para representación y brevedad). Biodentine ${ }^{\circledR}$ fue desarrollado deseando que fuera un elemento de reemplazo de dentina, una novedosa aplicación clínica de esta familia de materiales, con la intención de que funcionara a modo de una restauración coronal. ${ }^{4}$

Estos cementos poseen sustancias hidrófilas capaces de tolerar la humedad y pueden endurecer en presencia de fluidos biológicos (sangre, plasma, saliva, entre otros). Son mezclas aptas para liberar calcio y iones hidroxilo (actividad alcalinizante) en los líquidos circundantes, creando las condiciones para la formación de apatita. ${ }^{5}$

Por ello, los citados cementos tienen el potencial de aumentar la remineralización de lesiones de dentina previamente desmineralizadas y cariadas. Hoy en día, es posible solucionar diferentes problemas basándose

Tabla 3: Lista CONSORT de cementos basados con silicato de calcio A.

\begin{tabular}{|c|c|c|c|c|c|}
\hline Artículos & Trujillo, et al. & Daneshpoor, et al. & Salma Fathy & Li, et al. & Neves, et al. \\
\hline 1. Título y resumen & 1 & 1 & 1 & 1 & 1 \\
\hline 2. Trasfondo y objetivo & 1 & 1 & 1 & 1 & 1 \\
\hline 3. Diseño de prueba & 1 & 1 & 1 & 1 & 1 \\
\hline 4. Participantes & 1 & 1 & 1 & 1 & 1 \\
\hline 5. Intervenciones & 1 & 1 & 1 & 1 & 1 \\
\hline 6. Resultados & 1 & 1 & 1 & 1 & 1 \\
\hline 7. Tamaño muestral & 1 & 0 & 1 & 1 & 1 \\
\hline 8. Secuencia aleatoria & 1 & 1 & 1 & 1 & 1 \\
\hline 9. Asignación oculta & 0 & 0 & 0 & 0 & 1 \\
\hline 10. Implementación & 0 & 0 & 0 & 1 & 0 \\
\hline 11. Enmascaramiento & 0 & 0 & 0 & 1 & 1 \\
\hline 12. Métodos estadísticos & 1 & 1 & 1 & 1 & 1 \\
\hline 13. Flujo de participantes & 1 & 1 & 1 & 1 & 1 \\
\hline 14. Reclutamiento & 1 & 1 & 1 & 1 & 1 \\
\hline 15. Datos basales & 1 & 1 & 1 & 1 & 1 \\
\hline 16. Números analizados & 1 & 1 & 1 & 1 & 1 \\
\hline 17. Resultados y estimación & 1 & 1 & 1 & 1 & 1 \\
\hline 18. Análisis complementarios & 1 & 1 & 1 & 1 & 1 \\
\hline 19. Eventos adversos & 0 & 0 & 0 & 0 & 0 \\
\hline 20. Discusión/interpretación & 1 & 1 & 1 & 1 & 1 \\
\hline 21. Generalización & 1 & 1 & 1 & 1 & 1 \\
\hline 22. Balance Daño/beneficio & 1 & 1 & 1 & 1 & 1 \\
\hline Total & 18 & 17 & 18 & 20 & 20 \\
\hline
\end{tabular}

Fuente: directa. 
principalmente en el uso de materiales que en la actualidad existen en el mercado, tales como Biodentine ${ }^{\circledR}$ y TheraCal ${ }^{\circledR}$ o los cementos de silicato de calcio modificados biomiméticamente. ${ }^{6}$

Este cemento a base de silicato de calcio cristaliza cuando es mezclado con agua. Por medio de una reacción de hidratación del silicato tricálcico $\left(3 \mathrm{CaO} \bullet \mathrm{SiO}_{2}\right.$ $=\mathrm{C}_{3} \mathrm{~S}$ ), que produce un gel de silicato de calcio hidratado (CSH gel) e hidróxido de calcio $\left(\mathrm{Ca}(\mathrm{OH})_{2}\right)$. La hidratación del silicato tricálcico $\left(3 \mathrm{CaO} \bullet \mathrm{SiO}_{2}\right)$ conduce a la formación de un calcio hidratado hidróxido de silicato de gel e hidróxido de calcio $\left(\mathrm{Ca}(\mathrm{OH})_{2}\right){ }^{7}$

\section{FOSFATO DE CALCIO AMORFO}

La influencia de los productos lácteos (leche, concentrados lácteos y quesos) en la caries es conocida desde el año de 1980 cuando se demostró una acción anti- cariogénica tópica del queso. Dicho alcance protector se atribuyó a un efecto químico directo del contenido de fosfoproteína caseína y fosfato de calcio. El fosfopéptido de caseína es capaz de unir iones de calcio y fosfato formando así nanoclusters con fosfato de calcio amorfo. Estabilizados con fosfopéptido de caseína (CPP-ACP), ellos pueden mantener gradientes de alta concentración de iones de calcio y fosfato y pares de iones dentro de la lesión subsuperficial.

El aumento en la concentración de iones en el líquido de la abrasión da como resultado la formación de hidroxiapatita o fluorapatita a través del desarrollo de cristales, lo que deprime en gran medida la desmineralización del esmalte y mejora las condiciones de la remineralización. Se ha confirmado que, cuando los niveles adecuados de iones de calcio y fosfato se combinan con iones fluoruro (CPP-ACPF), la combinación puede provocar una remineralización significativa de los daños del esmalte.

Tabla 4: Lista CONSORT de cementos basados con silicato de calcio B.

\begin{tabular}{|c|c|c|c|c|}
\hline Artículos & Noha, et al. & Pires, et al. & Schwendicke, et al. & Suprastiwi, et al. \\
\hline 1. Título y resumen & 1 & 1 & 1 & 1 \\
\hline 2. Trasfondo y objetivo & 1 & 1 & 1 & 1 \\
\hline 3. Diseño de prueba & 1 & 1 & 1 & 1 \\
\hline 4. participantes & 1 & 1 & 1 & 1 \\
\hline 5. Intervenciones & 1 & 1 & 1 & 1 \\
\hline 6. Resultados & 1 & 1 & 1 & 1 \\
\hline 7. Tamaño muestral & 1 & 1 & 1 & 1 \\
\hline 8. Secuencia aleatoria & 1 & 1 & 1 & 0 \\
\hline 9. Asignación oculta & 0 & 1 & 0 & 0 \\
\hline 10. Implementación & 1 & 1 & 0 & 1 \\
\hline 11. Enmascaramiento & 0 & 1 & 0 & 0 \\
\hline 12. Métodos estadísticos & 1 & 1 & 1 & 1 \\
\hline 13. Flujo de participantes & 1 & 1 & 1 & 1 \\
\hline 14. Reclutamiento & 1 & 1 & 1 & 1 \\
\hline 15. Datos basales & 1 & 1 & 1 & 1 \\
\hline 16. Números analizados & 1 & 1 & 1 & 1 \\
\hline 17. Resultados y estimación & 1 & 1 & 1 & 1 \\
\hline 18. Análisis complementarios & 1 & 0 & 0 & 1 \\
\hline 19. Eventos adversos & 1 & 0 & 0 & 0 \\
\hline 20. Discusión/interpretación & 1 & 1 & 1 & 1 \\
\hline 21. Generalización & 1 & 1 & 1 & 1 \\
\hline 22. Balance Daño/beneficio & 1 & 1 & 1 & 1 \\
\hline Total & 20 & 20 & 17 & 18 \\
\hline
\end{tabular}

Fuente: directa. 
Tabla 5: Lista CONSORT de materiales a base de fosfopéptidos de caseína-fosfato de calcio amorfo A.

\begin{tabular}{lcccc}
\hline Artículos & Zhang, et al. & Vieira, et al. & Thierens, et al. & Soares, et al. \\
\hline 1. Título y resumen & 1 & 1 & 1 & 1 \\
2. Trasfondo y objetivo & 1 & 1 & 1 & 1 \\
3. Diseño de prueba & 1 & 1 & 1 & 1 \\
4. Participantes & 1 & 1 & 1 & 1 \\
5. Intervenciones & 1 & 1 & 1 & 1 \\
6. Resultados & 1 & 1 & 1 & 1 \\
7. Tamaño muestral & 1 & 1 & 1 & 1 \\
8. Secuencia aleatoria & 0 & 1 & 0 & 0 \\
9. Asignación oculta & 0 & 0 & 0 & 0 \\
10. Implementación & 0 & 0 & 0 & 0 \\
11. Enmascaramiento & 0 & 0 & 0 & 1 \\
12. Métodos estadísticos & 1 & 1 & 1 & 1 \\
13. Flujo de participantes & 1 & 1 & 1 & 1 \\
14. Reclutamiento & 1 & 1 & 1 & 1 \\
15. Datos basales & 1 & 1 & 1 & 1 \\
16. Números analizados & 1 & 1 & 1 & 1 \\
17. Resultados y estimación & 1 & 1 & 1 & 1 \\
18. Análisis complementarios & 1 & 1 & 1 & 1 \\
19. Limitaciones & 0 & 0 & 0 & 1 \\
20. Discusión/interpretación. & 1 & 1 & 1 & 18 \\
21. Generalización & 1 & 1 & 1 & 17 \\
22. Balance daño/beneficio & 1 & 1 & & \\
Total & 17 & & & 1 \\
\hline
\end{tabular}

Fuente: directa.

Se demostró que el fluoruro combinado con CPP-ACP se incorpora al cuerpo de la erosión de la mancha blanca y no se localiza en la capa superficial más externa del esmalte. La difusión profunda de iones de fluoruro junto con iones de calcio y fosfato en la ulceración permite un crecimiento sustancial de cristales (remineralización) en todo el cuerpo de la lesión. ${ }^{8}$

\section{ESTRATEGIA DE BÚSQUEDA}

La estrategia de búsqueda determinó inicialmente 691 publicaciones a base de silicato de calcio, 45 de los cuales se excluyeron por estar duplicados, luego se eliminaron 611 al aplicar los criterios de exclusión e inclusión. Los 35 artículos restantes fueron descargados y revisados en texto completo, eliminando 14 documentos por carecer de valor para este trabajo (Figura 1).

\section{SELECCIÓN DE ARTÍCULOS}

Los 21 documentos elegidos se publicaron en el periodo de los años 2013 a 2020. Estos manuscritos científicos fueron divulgados en diferentes revistas científicas (Tablas 1 y 2), así mismo la calidad de los artículos fue evaluada mediante la lista de chequeo CONSORT (Tablas 3 a 7), con 22 ítems a evaluar, siendo aceptados los que cumplieran con al menos 15 criterios. $^{9}$

\section{RESULTADOS}

\section{Dentina}

Se utilizaron diferentes técnicas queriendo evaluar la remineralización de la dentina: escaneo y microscopia electrónica de transmisión (SEM y TEM), espectroscopia 
infrarroja por transformada de Fourier (FTIR), espectroscopia Raman, difracción de rayos X (XRD), espectroscopia de rayos $X$ dispersiva de energía (EDX), microrradiografía, escaneo micro-CT (Tabla 8), determinándose que Biodentine ${ }^{\circledR}$ fue el material más utilizado (88\%) para realizar los estudios, el cual fue comparado en ese mismo porcentaje con otros elementos originados por silicato de calcio, alcanzando Biodentine ${ }^{\circledR}$ una diferencia significativa y mejores resultados con respecto a los otros cementos.

\section{Esmalte}

Se utilizaron variadas técnicas a fin de estimar la remineralización del esmalte, escaneo y microscopia electrónica de transmisión (Scanning Electron Microscope [SEM]) y (Transmission electron microscopy [TEM]), electroforesis, microrradiografía transversal, evaluación de fluorescencia cuantitativa inducida por la luz (QLF), valoración clínica, Diagnodent, microscopia de escaneo de láser confocal (CLSM), prueba de microdureza $(\mathrm{VHN})$, microdureza de superficie $(\mathrm{SMH})$, rugosidad de superficie (Ra), microscopia de fuerza atómica (AFM), difractómetro de rayos $X$ (DRX), microsonda electrónica (EPMA), y nanoindentación (Tabla 9), determinándose que, en $33.3 \%$ del total de los estudios revisados, el CCP-ACP de manera aislada exhibió un efecto remineralizante significativo, y en $25 \%$ el CCP-ACP combinado con el flúor arrojó este mismo resultado; en contraparte, $33.3 \%$ del conjunto de los documentos explorados señaló a otros materiales con una acción remineralizante más significativa con respecto al CCP-ACP y CCP-ACPF, igualmente, $8.3 \%$ del volumen de los manuscritos examinados no estableció ningún alcance remineralizante por parte del CCP-ACP.

Tabla 6: Lista CONSORT de materiales a base de fosfopéptidos de caseína-fosfato de calcio amorfo B.

\begin{tabular}{|c|c|c|c|c|}
\hline Artículos & Singh (2016) & Juárez (2014) & Zheng (2017) & Al-Batayneh, et al. (2017) \\
\hline 1. Título y resumen & 1 & 1 & 1 & 1 \\
\hline 2. Trasfondo y objetivo & 1 & 1 & 1 & 1 \\
\hline 3. Diseño de prueba & 1 & 1 & 1 & 1 \\
\hline 4. Participantes & 1 & 1 & 1 & 1 \\
\hline 5. Intervenciones & 1 & 1 & 1 & 1 \\
\hline 6. Resultados & 1 & 1 & 1 & 1 \\
\hline 7. Tamaño muestral & 1 & 1 & 1 & 1 \\
\hline 8. Secuencia aleatoria & 1 & 0 & 0 & 0 \\
\hline 9. Asignación oculta & 1 & 1 & 0 & 0 \\
\hline 10. Implementación & 1 & 1 & 0 & 0 \\
\hline 11. Enmascaramiento & 1 & 1 & 0 & 0 \\
\hline 12. Métodos estadísticos & 1 & 1 & 1 & 1 \\
\hline 13. Flujo de participantes & 1 & 1 & 1 & 1 \\
\hline 14. Reclutamiento & 1 & 1 & 1 & 1 \\
\hline 15. Datos basales & 1 & 1 & 1 & 1 \\
\hline 16. Números analizados & 1 & 1 & 1 & 1 \\
\hline 17. Resultados y estimación & 1 & 1 & 1 & 1 \\
\hline 18. Análisis complementarios & 1 & 0 & 1 & 1 \\
\hline 19. Limitaciones & 1 & 0 & 0 & 1 \\
\hline 20. Discusión/interpretación & 1 & 1 & 1 & 1 \\
\hline 21. Generalización & 1 & 1 & 1 & 1 \\
\hline 22. Balance daño/beneficio & 1 & 1 & 1 & 1 \\
\hline Total & 22 & 19 & 17 & 18 \\
\hline
\end{tabular}

Fuente: directa. 


\section{Tabla 7: Lista CONSORT de materiales a base de fosfopéptidos de caseína-fosfato de calcio amorfo $\mathrm{C}$.}

\begin{tabular}{lcccc}
\hline Artículos & Thabitha (2016) & Elkassas (2014) & Mohd (2014) & Zhou (2014) \\
\hline 1. Título y resumen & 1 & 1 & 1 & 1 \\
2. Trasfondo y objetivo & 1 & 1 & 1 & 1 \\
3. Diseño de prueba & 1 & 1 & 1 & 1 \\
4. Participantes & 1 & 1 & 1 & 1 \\
5. Intervenciones & 1 & 1 & 1 & 1 \\
6. Resultados & 1 & 1 & 1 & 1 \\
7. Tamaño muestral & 1 & 1 & 1 & 1 \\
8. Secuencia aleatoria & 1 & 0 & 1 & 0 \\
9. Asignación oculta & 0 & 0 & 0 & 0 \\
10. Implementación & 1 & 0 & 0 & 0 \\
11. Enmascaramiento & 0 & 0 & 0 & 1 \\
12. Métodos estadísticos & 1 & 1 & 1 & 1 \\
13. Flujo de participantes & 1 & 1 & 1 & 1 \\
14. Reclutamiento & 1 & 1 & 1 & 1 \\
15. Datos basales & 1 & 1 & 1 & 1 \\
16. Números analizados & 1 & 1 & 1 & 1 \\
17. Resultados y estimación & 1 & 1 & 1 & 0 \\
18. Análisis complementarios & 0 & 1 & 1 & 1 \\
19. Limitaciones & 0 & 0 & 0 & 1 \\
20. Discusión/interpretación & 1 & 1 & 1 & 1 \\
21. Generalización & 1 & 1 & 1 & 17 \\
22. Balance daño/beneficio & 1 & 1 & 18 & \\
Total & 18 & 17 & & 1 \\
\hline
\end{tabular}

Fuente: directa.

\section{Tabla 8: Instrumentos, material y duración de los estudios (cementos a base de silicato de calcio).}

\begin{tabular}{|c|c|c|c|}
\hline Autores & Instrumentos utilizados & Material analizado & Duración/ periodos \\
\hline Daneshpoor, et al. & $\begin{array}{l}\text { Espectroscopia (FTIR) y microscopia } \\
\text { electrónica de barrido (SEM) }\end{array}$ & $\begin{array}{l}\text { Biodentine }^{\circledR} \\
\text { TheraCal LC } \\
\text { CPP-ACP }\end{array}$ & Siete días \\
\hline Salma Fathy & $\begin{array}{c}\text { Espectroscopia de rayos X (EDX) } \\
\text { Microscopia electrónica de barrido (SEM) }\end{array}$ & $\begin{array}{l}\text { Biodenine }^{\circledR} \\
\text { TheraCal LC }\end{array}$ & $\begin{array}{l}\text { Una semana } \\
3 \text { y } 6 \text { meses }\end{array}$ \\
\hline $\mathrm{Li}$, et al. & $\begin{array}{c}\text { Microanálisis de sonda de electrones de } \\
\text { pistola de emisión de campo (Feg-EPMA), } \\
\text { espectroscopia Raman }\end{array}$ & $\begin{array}{l}\text { Biodentine }^{\circledR} \\
\text { MTA ProRoot } \\
\text { TheraCal LC }\end{array}$ & $\begin{array}{l}\text { Una semana } \\
1,3 \text { y } 6 \text { meses }\end{array}$ \\
\hline Neves, et al. & Micro CT & $\begin{array}{c}\text { Ionómero de vidrio, cemento Portland, } \\
\text { Biodentine }{ }^{\circledR}\end{array}$ & 30 días \\
\hline Noha, et al. & $\begin{array}{l}\text { Espectroscopia de rayos X, micro dureza } \\
\text { (VMH) }\end{array}$ & $\begin{array}{l}\text { Cemento a base de silicato de calcio, } \\
\text { cemento a base de hidróxido de calcio }\end{array}$ & 1,6 y 12 semanas \\
\hline Pires, et al. & Micro CT & $\begin{array}{l}\text { Policarboxilato de Zinc, MTA, } \\
\text { Biodentine }^{\circledR} \text {, GI (3M ESPE) }\end{array}$ & 30 días \\
\hline
\end{tabular}


Continúa Tabla 8: Instrumentos, material y duración de los estudios (cementos a base de silicato de calcio).

\begin{tabular}{|c|c|c|c|}
\hline Autores & Instrumentos utilizados & Material analizado & Duración/ periodos \\
\hline Schwendicke, et al. & $\begin{array}{l}\text { Microrradiografía transversal, micro dureza, } \\
\text { microscopia electrónica de transmisión }\end{array}$ & $\begin{array}{c}\text { Biodentine }{ }^{\circledR} \text { MTA, adhesivo } \\
\text { experimental }\end{array}$ & Tres meses \\
\hline \multirow[t]{2}{*}{ Suprastiwi, et al. } & Microscopia electrónica de transmisión & Biodentine $^{\circledR}$ & 14 días \\
\hline & & Resina compuesta & \\
\hline \multirow[t]{4}{*}{ Trujillo, et al. } & Microscopia electrónica de barrido y & Biodentine ${ }^{\circledR}$ MTA & 20 días \\
\hline & espectroscopia Raman & $\begin{array}{c}\text { Repair } \\
\mathrm{HP}^{\circledR}\end{array}$ & \\
\hline & & MTAA & \\
\hline & & $\operatorname{IRM}^{\circledR}$ & \\
\hline
\end{tabular}

Fuente: directa.

FTIR = espectroscopia infrarroja por transformada de Fourier, SEM = Scanning Electron Microscope, CPP-ACP = Fosfopéptidos de caseína-fosfato de calcio amorfo, EDX = Espectroscopia de rayos X dispersiva de energía, Feg-EPMA = Microanálisis de sonda de electrones de pistola de emisión de campo, MTA = Mineral de trióxido agregado, Micro CT = Micro-computed tomography, VMH = Vickers Microhardness, $\mathrm{HP}^{\circledR}=$ En referencia al Mineral de trióxido agregado Repair HP ${ }^{\circledR}$, MTAA = En referencia al Mineral de trióxido agregado Angelus ${ }^{\circledR}$, IRM ${ }^{\circledR}=$ En referencia al grupo control negativo IRM ${ }^{\circledR}$.

\section{Tabla 9: Instrumentos, material y duración de los estudios (CCP-ACP).}

\begin{tabular}{|c|c|c|c|}
\hline Autores & Instrumentos o métodos & Materiales usados & Duración/ periodos \\
\hline Zhang, et al. & $\begin{array}{l}\text { Electroforesis, tinción con hematoxilina } \\
\text { eosina }\end{array}$ & $\begin{array}{l}\text { Fosfopéptidos de caseína-fosfato de calcio } \\
\text { amorfo (CCP-ACP) }\end{array}$ & Cinco horas \\
\hline Vieira, et al. & Tomografía computarizada sincrotrón & CCP-ACP, gel neutro de NaF al 2 \% & 3 y 5 horas \\
\hline Thierens, et al. & Microrradiografía transversal & $\begin{array}{l}\text { CСР-АСР } \\
\text { CСР-АCРF }\end{array}$ & 6 y 12 semanas \\
\hline Soares, et al. & $\begin{array}{l}\text { Microscopio electrónico de Barrido } \\
\text { (SEM), máquina de prueba de } \\
\text { microdureza (VMT) }\end{array}$ & $\begin{array}{c}\text { CCP-ACPF } \\
\text { Vidrio Bioactivo (SHY-NM) } \\
\text { Gel de Hidroxiapatita mejorado con flúor } \\
\text { (ReminPro), Péptido autoensamblable (P11-4) }\end{array}$ & Un mes \\
\hline Singh, et al. & Evaluación clínica, Diagnodent & $\begin{array}{c}\text { Pasta dental con flúor, CPP-ACP más crema } \\
\text { de remineralización }\end{array}$ & 1,3 y 6 meses \\
\hline Juarez, et al. & Diagnodent. Mod. 2095 & $\begin{array}{c}\text { CPP-ACPF } \\
\text { Gel de fluoruro acidulado al } 2 \%\end{array}$ & Seis meses \\
\hline $\begin{array}{l}\text { Al-Batayneh, } \\
\text { et al. }\end{array}$ & $\begin{array}{l}\text { Evaluación de fluorescencia cuantitativa } \\
\text { inducida por la luz (QLF) }\end{array}$ & $\begin{array}{c}\text { Fluoruro } \\
\text { CCP-ACP } \\
\text { Fluoruro seguido de CCP-ACP } \\
\text { CCP-ACP seguido de fluoruro }\end{array}$ & 10 semanas \\
\hline Zheng, et al. & $\begin{array}{l}\text { Microscopia de escaneo de laser confocal } \\
\text { (CLSM), Microscopia Electrónica de barrido }\end{array}$ & СРР-АСР & 12 horas \\
\hline Thabitha, et al. & $\begin{array}{c}\text { Prueba de microdureza (VHN), microscopia } \\
\text { Electrónica de barrido }\end{array}$ & $\begin{array}{l}\text { Fosfato de sacarosa (CaSP), fosfato de calcio, } \\
\text { CPP-ACP }\end{array}$ & 12 días \\
\hline Elkassas, et al. & $\begin{array}{l}\text { Microdureza de superficie }(\mathrm{SMH}) \text {, } \\
\text { rugosidad de superficie }(\mathrm{Ra}), \\
\text { microscopia electrónica de barrido }\end{array}$ & $\begin{array}{c}\text { Barniz blanco Clinpro } \\
\text { Fosfato de calcio amorfo (ACPF), Tooth Mousse } \\
\text { Plus }^{\mathrm{TM}}(\mathrm{CPP}-\mathrm{ACPF}), \text { Vanish TMXT }\end{array}$ & 2 y 4 semanas \\
\hline
\end{tabular}




\section{Continúa Tabla 9: Instrumentos, material y duración de los estudios (CCP-ACP).}

\begin{tabular}{|c|c|c|c|}
\hline Autores & Instrumentos o métodos & Materiales usados & Duración/ periodos \\
\hline Zhou, et al. & $\begin{array}{c}\text { Microscopia de fuerza atómica (AFM), } \\
\text { difractómetro de rayos X (DRX), } \\
\text { microsonda electrónica (EPMA), } \\
\text { nanoindentación }\end{array}$ & $\begin{array}{c}\text { Agua des ionizada } \\
\text { CCP-ACP } \\
\text { NaF }\end{array}$ & 4, 8, 12 y 24 horas \\
\hline Mohd, et al. & $\begin{array}{l}\text { Microdureza superficial Knoop (KHN) } \\
\text { y microrradiografía transversal (TMR) }\end{array}$ & $\begin{array}{c}\text { Duraphat }^{\circledR}(\mathrm{NaF}) \\
\text { MI Varnish } \\
\text { (NaF, CPP-ACP) } \\
\text { Embrace Varnish }^{\mathrm{TM}} \\
(\mathrm{NaF}, \mathrm{CXP}) \\
\text { Enamel Pro Varnish }^{\circledR} \\
\text { (NaF, ACP) } \\
\text { Clinpro White Varnish TM (NaF, TCP) }\end{array}$ & Ocho días \\
\hline
\end{tabular}

Fuente: directa.

CCP-ACP = Fosfopéptidos de caseína-fosfato de calcio amorfo, CCP-ACPF = Fosfopéptidos de caseína-fosfato de calcio amorfo adicionado con flúor, SHY-NM = En referencia al vidrio bioactivo del Grupo Farmacéutico Novamind (SHY-NM, Group Pharmaceuticals Ltd., India), NaF, CPP-ACP = En referencia a los barnices de fluoruro tópico que contienen fluoruro de sodio (MI Varnish ${ }^{\mathrm{TM}}$, $\mathrm{NaF}, \mathrm{CXP}=$ En referencia a los barnices de fluoruro tópico que contienen fosfato y calcio recubiertos de xilitol (Embracetm Varnish), NaF, ACP = En referencia a los barnices de fluoruro tópico que contienen fosfato de calcio amorfo (Enamel Pro Varnish), NaF, TCP = En referencia al barniz blanco Clinprotm fosfato tricálcico (TCP).

\section{REFERENCIAS}

1. Giani A, Cedrés C. Avances en protección pulpar directa con materiales bioactivos. Actas Odontológicas. 2017; 14 (1): 4-13.

2. Cedillo VJJ, Cedillo FJE. Protocolo clínico actual para restauraciones profundas. ADM. 2013; 70 (5): 263-275.

3. Ameneiros NO, Gamboa SJ, Soto RA, Martínez BA, Ruiz CH. El uso de materiales bioactivos en la estomatología conservadora contemporánea. Biodentine ${ }^{\circledR}$. Invest Medicoquir. 2019; 11 (3).

4. Watson TF, Atmeh AR, Sajini S, Cook RJ, Festy F. Present and future of glass-ionomers and calcium-silicate cements as bioactive materials in dentistry: biophotonics-based interfacial analyses in health and disease. Dent Mater. 2014; 30 (1): 50-61.

5. Garchitorena FMI. Materiales bioactivos en la remineralización dentinaria. Odontoestomatología. 2016; 18 (28): 11-19.

6. Prati C, Gandolfi MG. Calcium silicate bioactive cements: Biological perspectives and clinical applications. Dent Mater. 2015; 31 (4): 351-370.

7. Hincapié NS, Valerio RAL. Biodentine: a new material for pulp therapy. Universitas Odontologica, págs: 2015; 34 (73): 69-76.
8. Thierens LAM, Moerman S, Elst CV, Vercruysse C, Maes P, Temmerman $\mathrm{L}$ et al. The in vitro remineralizing effect of CPP-ACP and CPP-ACPF after 6 and 12 weeks on initial caries lesion. J Appl Oral Sci. 2019; 27: e20180589.

9. Moher D, Hopewell S, Schulz KF, Montori V, Gotzsche PC, Devereaux PJ et al. CONSORT 2010 explanation and elaboration: updated guidelines for reporting parallel group randomized trials. BMJ. 2010; 340: c869.

Correspondencia:

Ian Michel Jiménez Rosas

E-mail: ianmicheljimenezrosas@gmail.com

Conflicto de intereses: El autor declara no tener ningún conflicto de intereses.

Financiamiento: Ninguno. 